\title{
RESEARCH
}

Open Access

\section{Left ventricular function recovery in peripartum cardiomyopathy: a cardiovascular magnetic resonance study by myocardial T1 and T2 mapping}

Yao-Dan Liang ${ }^{1,2,3+}$, Yuan-Wei Xu' ${ }^{1+}$, Wei-Hao Li ${ }^{1}$, Ke Wan ${ }^{4}$, Jia-Yu Sun ${ }^{5}$, Jia-Yi Lin ${ }^{1}$, Qing Zhang ${ }^{1}$, Xiao-Yue Zhou ${ }^{6}$ and Yu-Cheng Chen ${ }^{1 *}$ (D)

\begin{abstract}
Background: Peripartum cardiomyopathy (PPCM) is rare and potentially life-threatening; its etiology remains unclear. Imaging characteristics on cardiovascular magnetic resonance (CMR) and their prognostic significance have rarely been studied. We sought to determine CMR's prognostic value in PPCM by using T1 and T2 mapping techniques.

Methods: Data from 21 PPCM patients from our CMR registry database were analyzed. The control group comprised 20 healthy age-matched females. All subjects underwent comprehensive contrast-enhanced CMR. T1 and T2 mapping using modified Look-Locker inversion recovery and T2 prep balanced steady-state free precession sequences, respectively. Ventricular size and function, late gadolinium enhancement (LGE), myocardial T1 value, extracellular volume (ECV), and T2 value were analyzed. Transthoracic echocardiography was performed at baseline and during follow-up. The recovered left ventricular ejection fraction (LVEF) was defined as LVEF $\geq 50 \%$ on echocardiography follow-up after at least 6 months of the diagnosis.

Results: CMR imaging showed that the PPCM patients had severely impaired LVEF and right ventricular ejection fraction (LVEF: $26.8 \pm 10.6 \%$; RVEF: $33.9 \pm 14.6 \%$ ). LGE was seen in eight (38.1\%) cases. PPCM patients had significantly higher native T1 and ECV (1345 \pm 79 vs. $1212 \pm 32 \mathrm{~ms}, P<0.001 ; 33.9 \pm 5.2 \%$ vs. $27.1 \pm 3.1 \%, P<0.001$; respectively $)$ and higher myocardial T2 value $(42.3 \pm 3.7$ vs. $36.8 \pm 2.3 \mathrm{~ms}, P<0.001)$ than did the normal controls. After a median 2.5 -year follow-up (range: 8 months-5 years), six patients required readmission for heart failure, two died, and 10 showed left ventricular function recovery. The LVEF-recovered group showed significantly lower ECV (30.7 $\pm 2.1 \%$ vs. $36.8 \pm 5.6 \%$, $P=0.005)$ and $\mathrm{T} 2(40.6 \pm 3.0$ vs. $43.9 \pm 3.7 \mathrm{~ms}, P=0.040)$ than the unrecovered group. Multivariable logistic regression analysis showed ECV $(\mathrm{OR}=0.58$ for per $1 \%$ increase, $P=0.032)$ was independently associated with left ventricular recovery in PPCM.
\end{abstract}

Conclusions: Compared to normal controls, PPCM patients showed significantly higher native T1, ECV, and T2. Native $\mathrm{T} 1, \mathrm{ECV}$, and T2 were associated with LVEF recovery in PPCM. Furthermore, ECV could independently predict left ventricular function recovery in PPCM.

Keywords: Peripartum cardiomyopathy, Cardiovascular magnetic resonance, Extracellular volume, T1 mapping, T2 mapping, Left ventricular function recovery

\footnotetext{
* Correspondence: chenyucheng2003@126.com

${ }^{\dagger}$ Yao-Dan Liang and Yuan-Wei Xu contributed equally to this work.

'Department of Cardiology, West China Hospital, Sichuan University, No.37,

Guo Xue Xiang, Chengdu, Sichuan 610041, People's Republic of China

Full list of author information is available at the end of the article
}

(c) The Author(s). 2019 Open Access This article is distributed under the terms of the Creative Commons Attribution 4.0 International License (http://creativecommons.org/licenses/by/4.0/), which permits unrestricted use, distribution, and 


\section{Background}

Peripartum cardiomyopathy (PPCM) is a rare, lifethreatening pregnancy-associated disease that is typically marked by ventricular dysfunction. The incidence of PPCM is gradually increasing [1] and varies geographically, most likely due to socioeconomic and genetic factors [2]. Previous studies have reported the incidence rates of the disease in different geographical regions as follows: 1 per 300 live births in Haiti [3], 1 per 346 live births in China [4], 1 per 1000 live births in South Africa [5], and 1 per 900-4000 live births in the United States [6-8]. Although viral myocarditis, abnormal immune responses to pregnancy, abnormal responses to hemodynamic stress during pregnancy, cytokine-induced inflammation, and genetic factors have been suggested to be involved in the pathogenesis of PPCM, the precise pathogenetic mechanism remains unclear [9]. The reported outcomes vary from recovery of the left ventricular (LV) ejection fraction (LVEF) within 6 months of diagnosis in $73 \%$ of the study patients [10] to the need for heart transplantation or even death $[11,12]$. The lack of prognostic indicators in PPCM patients is another important clinical challenge [13].

Cardiovascular magnetic resonance imaging (CMR) is a recognized radiation-free technique for assessing cardiac structure and function and myocardial tissue characterization. Several case series and reports have shown that CMR late gadolinium enhancement (LGE) has potential value in predicting poor clinical outcomes in PPCM patients $[11,14,15]$. However, two recent multicenter and nationwide studies showed that LGE at the baseline and during follow-up were uncommon (only 3.6$14 \%)$ in PPCM $[16,17]$. Furthermore, patients who did not have LGE also showed poor clinical outcomes [18]. This suggests that LGE alone has limitations as a prognostic indicator in PPCM patients. Furthermore, two case series showed myocardial edema by using T2-weighted images in PPCM patients [18, 19], while another case series showed no obvious edema on T2-weighted images [14]. This may be due to the inherent limitations of T2weighted imaging or poor image quality [20]. CMR with native $\mathrm{T} 1$ and $\mathrm{T} 2$ mapping offers a unique noninvasive way to quantify myocardial diffused fibrosis, measure extracellular volume (ECV), and detect myocardial edema. A recent CMR study with T1 mapping showed that LV remodeling is associated with myocardial hypertrophy, but not with edema or diffuse fibrosis of the myocardium or LV contractile dysfunction during normal pregnancy [21]. However, no study has analyzed myocardial tissue characterization in PPCM by native T1 and T2 mapping or its predictive value for outcomes in PPCM patients. Therefore, the objective of our study was to analyze myocardial tissue characterization on CMR with native T1 and T2 mapping techniques and determine their potential value for predicting $\mathrm{LV}$ function recovery.

\section{Methods}

\section{Study population}

During the period from August 2013 to June 2018, 21 new-onset PPCM patients (presence of typical heart failure (HF) symptoms $\leq 6$ months) in our CMR registry nonischemic cardiomyopathy cohort were included. No patient had a history of cardiovascular disease before developing PPCM. No abnormality had been found in the electrocardiogram (ECG) or physical examination during pre-pregnancy examination. The onset times for abnormalities ranged from the last month up prior to delivery to 5 months postpartum and fit into the strict criteria of PPCM established by the European Society of Cardiology [13]. All patients underwent CMR scan after the termination of pregnancy. The interval between CMR and onset time ranged from 7 days to 3 months, with a median of 1 month. Follow-up was performed by a review of the electronic databases and telephone interviews. An LVEF of $\geq 50 \%$ by transthoracic echocardiography follow-up after a minimum of 6 months was defined as LV function recovery. Ten of the enrolled PPCM patients underwent repeated CMR imaging during the follow-up. We identified 20 age-matched healthy females, without a history of hypertension, diabetes, or any other known heart disease, as the control group.

\section{CMR data acquisition \\ Scanning protocol}

CMR examination was performed with a standardized clinical protocol [22] using a $3 \mathrm{~T}$ CMR scanner (MAGNTEOM Trio, Siemens Healthineers, Erlangen, Germany) with an eight-channel phased-array body coil. All images were acquired with ECG-gating and breath-holding. The imaging protocol consisted of a balanced steady-state free precession (bSSFP) cine sequence for left and right ventricular function, an inversion recovery turbo-flash pulse sequence after gadolinium administration for LGE, a modified LookLocker inversion recovery (MOLLI) sequence for native T1 mapping, and a T2 mapping sequence using T2 prep with bSSFP for myocardial edema. Cine images were acquired in three long axes (2-, 3-, and 4chamber) and consecutive short-axis views covering the $\mathrm{LV}$ from the base to the apex $(\mathrm{TR}=3.4 \mathrm{~ms}, \mathrm{TE}=$ $1.3 \mathrm{~ms}$, flip angle $50^{\circ}$, FOV $320 \times 340 \mathrm{~mm}^{2}$, matrix size $256 \times 144$, slice thickness $8 \mathrm{~mm}$ with no gap). LGE images were acquired $15 \mathrm{~min}$ after intravenous administration of $0.15 \mathrm{mmol} / \mathrm{kg}$ gadopentetate dimeglumine (Bayer Healthcare, Berlin, Germany) by using the inversion recovery technique in the identical views $\left(\mathrm{TR}=700 \mathrm{~ms}, \quad \mathrm{TE}=1.56 \mathrm{~ms}\right.$, flip angle $20^{\circ}$, matrix $256 \times 144)$. The inversion time (TI) was individually optimized to null the normal myocardial signal by using a TI scout sequence. 


\section{T1 mapping}

T1 mapping was acquired in three short-axis slices (basal, mid, and apical) by using a MOLLI sequence (Siemens Healthineers, works-in-progress method 448) with a fixed 5(3)3/4(1)3(1)2 sampling scheme for pre- and post-contrast mapping, respectively. The parameters were as follows: non-selective inversion pulse, bSSFP single-shot readout with $35^{\circ}$ excitation flip angle, minimum inversion recovery time of $100 \mathrm{~ms}$, inversion recovery time increment of $80 \mathrm{~ms}$, TR/TE of $2.5 / 1.12 \mathrm{~ms}$, FOV of $360 \times 272 \mathrm{~mm}^{2}$, matrix size of $256 \times 144$, and slice thickness of $8 \mathrm{~mm}$. Post-contrast T1 mapping was performed $20 \mathrm{~min}$ after the injection of gadolinium. The T1 map was generated inline right after each scan.

\section{T2 mapping}

Data were acquired from LV basal, mid, and apical short-axis slices using the $\mathrm{T}_{2}$-prepared single-shot bSSFP technique before the administration of gadolinium. Three single-shot bSSFP images with different $\mathrm{T}_{2}$ preparation times $\left(\mathrm{TE}_{\mathrm{T} 2 \mathrm{P}}=0 \mathrm{~ms}, 25 \mathrm{~ms}, 55 \mathrm{~ms}\right)$ were obtained at end-diastole during one breath-hold. Imaging parameters were as follows: $\mathrm{TR}=2.4 \mathrm{~ms}, \mathrm{TE}=1.0 \mathrm{~ms}$, $\mathrm{FA}=70^{\circ}, \quad \mathrm{FOV}=320-340 \times 262-278 \mathrm{~mm}^{2}$, matrix of $176 \times 144$, slice thickness of $8 \mathrm{~mm}, \mathrm{BW}=1093 \mathrm{~Hz} / \mathrm{px}$, and GRAPPA acceleration factor of 2 . An optional fast variational non-rigid image registration algorithm was used to compensate for the in-plane motion between images. Finally, a pixel-wise myocardial T2 map was generated inline by using curve-fitting with a twoparameter equation.

\section{Imaging analysis}

Two cardiovascular imaging physicians blinded to clinical data independently analyzed all CMR images. LV and right ventricular (RV) volumes and LVEF and RV ejection fraction (RVEF) were assessed using dedicated commercial CMR software (Medis Suite, Version 3.1, Medis, Leiden, Netherlands). Contouring of ventricles was performed following a standardized protocol according to the Society for Cardiovascular Magnetic Resonance (SCMR) post-processing guidelines [23]. Papillary muscles and trabeculations were excluded from the myocardial mass and included in the ventricular volume. Images were evaluated for myocardial LGE by two experienced CMR readers (LYD with 3 years' clinical experience, CYC with 8 years' clinical experience). T1 and T2 mapping images were analyzed using dedicated software (Medis Suite, Version 3.1). Myocardial T1 mapping was measured on the mid-ventricular LV short-axis slice. A few contour corrections were required to exclude epicardial fat and trabeculations or blood pool on the images. The T1 value of the blood pool was determined by manually drawing a region of interest in the LV cavity.
The hematocrit (HCT) was obtained on the same day as CMR in normal controls and within 3 days prior to the scanning in patients. It was used to calculate the ECV according to the following equation: $\mathrm{ECV}=(1$ $\mathrm{HCT}) \times\left(\Delta \mathrm{R} 1_{\text {myocardium }} / \Delta \mathrm{R} 1_{\text {blood }}\right)$ [24]. T2 mapping was measured on the same mid-ventricular LV slice as T1 mapping. The LV myocardium was manually contoured by tracing the endocardial and epicardial borders. Trabeculations and epicardial fat were carefully excluded, and only the myocardium was included for analysis.

\section{Statistical analysis}

Kolmogorov-Smirnov tests were used to check continuous variables for normal distribution. Normally distributed variables were described as mean \pm SD values and tested with Student's t tests. Non-normally distributed variables were described as median and quartile values and tested with Mann-Whitney U tests. Categorical variables were described as frequencies and percentiles and tested with Pearson's Chi-square test or Fisher's exact tests. A Pearson correlation coefficient was used to analyze the correlations between continuous variables. The area under the curve (AUC) in the receiver operating characteristic (ROC) analysis for LVEF recovery was estimated to determine the optimal cutoff points for ECV and native $\mathrm{T} 1$ and $\mathrm{T} 2$ to predict LVEF recovery. The cutoff value was defined as the value with the maximum Youden index. Univariate and multivariate Logistic regression analyses were performed to identify indicators associated with LV recovery in PPCM patients. Variables with a $P$ value $<0.10$ (except for diastolic blood pressure, to avoid the collinearity with systolic blood pressure) in univariate analyses were entered into the multivariate logistic regression model. They were analyzed by the forward stepwise method with entry and removal of $p$-values of 0.05 and 0.10 , respectively. The results obtained using the logistic regression models were presented as odds ratios (ORs) and 95\% confidence intervals (95\% CIs). A major adverse cardiac event (MACE) was defined as readmission for HF or cardiac death. Survival curves for MACEs were plotted with the Kaplan-Meier analysis according to the ECV and compared with the results of the log-rank test. In the subgroup analysis of 10 patients who underwent repeated CMR imaging, comparison of tissue characteristics at follow-up and baseline CMR findings was performed using the paired T-test. A $P$ value $<0.05$ was considered to indicate statistical significance. All statistical analyses were performed using standard statistical software (SPSS Statistics, Version 24.0, Statistical Package for the Social Sciences, International Business Machines, Inc., Armonk, New York, USA). 


\section{Results}

\section{Clinical characteristics}

During the study period, 21 PPCM patients (28.4 \pm 5.9 years) were included in our CMR registry database. Among these, 15 patients were primiparas and one had twin fetuses. All had obvious symptoms of HF accompanied by an increased serum N-terminal pro-B-type natriuretic peptide (NT-proBNP) level. Their symptom onset times ranged from the last month up to 5 months postpartum. None had a history of hypertension, preeclampsia, or eclampsia before or during the pregnancy. Seventeen patients underwent a cesarean delivery in this gestation, while the others gave natural birth. All patients presented with a sinus rhythm and only one patient showed a left bundle branch block on ECG. Echocardiography showed an LVEF less than $45 \%$ in all patients at the time of diagnosis and a mural thrombus in two patients. In comparison with 20 age-matched normal female patients, the PPCM patients had lower systolic blood pressure $(103 \pm 9$ vs $121 \pm 6 \mathrm{mmHg}, P<0.001)$ and a higher heart rate $(86 \pm 17$ vs $77 \pm 10 \mathrm{bpm}, P=$ 0.042) (Table 1). All PPCM patients were given guideline-directed medical therapy, which included a loop diuretic, angiotensin-converting enzyme inhibitor (ACEI)/angiotensin II receptor blockers (ARB), $\beta$ blocker, and antisterone (Table 2).

\section{CMR imaging characteristics}

PPCM patients demonstrated a larger LV end diastolic volume index (LVEDVI, $155 \pm 32$ vs. $75 \pm 12 \mathrm{ml} /$ $\mathrm{m}^{2}$ and RV end-diastolic volume index (RVEDVI, $121 \pm 41$ vs. $70 \pm 18 \mathrm{ml} / \mathrm{m}^{2}$, both $p<0.001$ ), higher $\mathrm{LV}$ mass index (LVMI, $73.6 \pm 16.4$ vs. $40.8 \pm 6.1 \mathrm{~g} / \mathrm{m}^{2}, p<$ $0.001)$, lower LVEF $(27 \pm 11 \%$ vs. $61 \pm 3 \%, p<0.001)$, and lower RVEF $(34 \pm 15 \%$ vs. $56 \pm 6 \%, p<0.001)$ in comparison with normal controls. LGE was identified in eight $(8 / 21,38 \%)$ PPCM patients, which included five patients with middle-line patterns, two with diffused patterns, and one with a patchy pattern. Among the 10 LVEF-recovered patients, two were LGEpositive with middle-line patterns, while the unrecovered patients included three with middle-line patterns, two with diffused patterns, and one with a patchy LGE pattern. In $\mathrm{T} 1$ and $\mathrm{T} 2$ mapping, the patient group showed higher values for ECV $(33.9 \pm 5.2 \%$ vs. $27.1 \pm 3.1 \%, \quad p<0.001)$, native T1 $(1345 \pm 79$ vs. $1212 \pm 32 \mathrm{~ms}, p<0.001)$ and T2 $(42.3 \pm 3.7$ vs. $36.8 \pm$ $2.3 \mathrm{~ms}, \quad P<0.001$ ) than the control group (Table 1 ). When data from the PPCM patients and normal groups were pooled, LVEF was negatively correlated with ECV (Pearson's $r=-0.714, p<0.001$ ), native T1 (Pearson's $r=-0.833, p<0.001$ ), and T2 (Pearson's $r=-0.649, p<0.001)$.
Table 1 Characteristics and CMR findings of PPCM patients and healthy volunteers

\begin{tabular}{|c|c|c|c|}
\hline & $\operatorname{PPCM}(n=21)$ & Normal $(n=20)$ & $P$ values \\
\hline Age, years & $28.4 \pm 5.9$ & $31.3 \pm 8.3$ & 0.207 \\
\hline Female, n (\%) & $21(100)$ & $20(100)$ & NA \\
\hline $\mathrm{BMI}\left(\mathrm{kg} / \mathrm{m}^{2}\right)$ & $22.1 \pm 4.0$ & $21.3 \pm 3.2$ & 0.522 \\
\hline $\mathrm{SBP}(\mathrm{mmHg})$ & $103.1 \pm 9.2$ & $121.1 \pm 6.3$ & $<0.001$ \\
\hline $\mathrm{DBP}(\mathrm{mmHg})$ & $68.1 \pm 10.5$ & $71.5 \pm 8.5$ & 0.269 \\
\hline HR (beat/min) & $86.3 \pm 16.5$ & $77.3 \pm 10.1$ & 0.042 \\
\hline NYHA class & & & NA \\
\hline$\|/\| I / I V, n$ & $3 / 11 / 7$ & - & \\
\hline \multicolumn{4}{|l|}{ Lab } \\
\hline $\mathrm{HCT}(\%)$ & $40.4 \pm 4.3$ & $41.8 \pm 3.1$ & 0.262 \\
\hline NT-proBNP (pg/ml) & $2189.0[904.0,3435.0]$ & - & NA \\
\hline $\mathrm{TnT}(\mathrm{ng} / \mathrm{L})$ & $10.3[4.7,16.1]$ & - & NA \\
\hline \multicolumn{4}{|l|}{ CMR findings } \\
\hline LVEDVI $\left(\mathrm{ml} / \mathrm{m}^{2}\right)$ & $154.7 \pm 31.6$ & $74.5 \pm 12.4$ & $<0.001$ \\
\hline LVEF (\%) & $26.8 \pm 10.6$ & $61.2 \pm 3.3$ & $<0.001$ \\
\hline LVMI $\left(\mathrm{g} / \mathrm{m}^{2}\right)$ & $73.6 \pm 16.4$ & $40.8 \pm 6.1$ & $<0.001$ \\
\hline RVEDVI $\left(\mathrm{ml} / \mathrm{m}^{2}\right)$ & $120.7 \pm 41.0$ & $70.1 \pm 18.1$ & $<0.001$ \\
\hline RVEF (\%) & $33.9 \pm 14.6$ & $56.4 \pm 5.9$ & $<0.001$ \\
\hline LGE, n (\%) & $8(38.1 \%)$ & 0 & NA \\
\hline ECV (\%) & $33.9 \pm 5.2$ & $27.1 \pm 3.1$ & $<0.001$ \\
\hline Native T1 (ms) & $1345 \pm 79$ & $1212 \pm 32$ & $<0.001$ \\
\hline Post T1 (ms) & $451 \pm 69$ & $483 \pm 49$ & 0.090 \\
\hline $\mathrm{T} 2$ (ms) & $42.3 \pm 3.7$ & $36.8 \pm 2.3$ & $<0.001$ \\
\hline
\end{tabular}

Female, NYHA class and LGE were described as frequency. NT-proBNP and TnT were described with medians and quartiles, while other data were presented as mean and standard deviations

Abbreviations: CMR cardiovascular magnetic resonance, $P P C M$ peripartum cardiomyopathy, $B M I$ body mass index, $S B P$ systolic blood pressure, $D B P$ diastolic blood pressure, HR heart rate, NYHA New York Heart Association, HCT hematocrit, NT-proBNP N-terminal pro-B-type natriuretic peptide, $T n T$ troponin $\mathrm{T}$, LVEDVI left ventricular end diastolic volume index, $L V E F$ left ventricular ejection fraction, $L V M I$ left ventricular mass index, RVEDVI right ventricular end diastolic volume index, RVEF right ventricular ejection fraction, LGE late gadolinium enhancement, ECV extracellular volume, NA not applicable

\section{CMR imaging characteristics and associations with patient outcome}

Ten (48\%) of the 21 PPCM patients showed LVEF recovery, defined by echocardiography as LVEF $\geq 50 \%$ over at least 6 months' follow-up. In comparison with the LVEFunrecovered group, the LVEF-recovered group showed a significantly lower baseline ECV $(30.7 \pm 2.1 \%$ vs. $36.8 \pm$ $5.6 \%, P=0.005)$, native T1 $(1301 \pm 71$ vs. $1385 \pm 66 \mathrm{~ms}$, $P=0.011)$, and T2 (40.6 \pm 3.0 vs. $43.9 \pm 3.7 \mathrm{~ms}, P=0.040)$ (Fig. 1). However, the LVEDVI, LVEF, LV mass index, RVEDVI, and RVEF at baseline did not show a significant difference between these two groups (all $P>0.3$ ). Furthermore, the unrecovered group showed a higher percentage of LGE than the recovered group, although 
Table 2 Comparison of CMR findings between LVEF-recovered and -unrecovered PPCM patients

\begin{tabular}{|c|c|c|c|}
\hline & $\begin{array}{l}\text { Recovered } \\
(n=10)\end{array}$ & $\begin{array}{l}\text { Unrecovered } \\
(n=11)\end{array}$ & $P$ values \\
\hline Age, years & $27.5 \pm 5.9$ & $29.3 \pm 6.0$ & 0.505 \\
\hline $\mathrm{BMI}\left(\mathrm{kg} / \mathrm{m}^{2}\right)$ & $22.2 \pm 2.3$ & $21.9 \pm 5.3$ & 0.852 \\
\hline $\mathrm{SBP}(\mathrm{mmHg})$ & $106.8 \pm 9.0$ & $99.7 \pm 8.5$ & 0.079 \\
\hline $\mathrm{DBP}(\mathrm{mmHg})$ & $73.4 \pm 12.1$ & $63.3 \pm 6.0$ & 0.023 \\
\hline HR (beat/min) & $89.2 \pm 18.7$ & $83.7 \pm 14.6$ & 0.461 \\
\hline NYHA class & & & 0.771 \\
\hline$\|/\| I / I V, n$ & $2 / 5 / 3$ & $1 / 6 / 4$ & \\
\hline \multicolumn{4}{|l|}{ Lab } \\
\hline $\mathrm{HCT}(\%)$ & $41.6 \pm 3.8$ & $39.5 \pm 4.6$ & 0.280 \\
\hline $\begin{array}{l}\text { NT-proBNP } \\
(\mathrm{pg} / \mathrm{ml})\end{array}$ & $\begin{array}{l}1451.5[470.0 \\
3398.5]\end{array}$ & $\begin{array}{l}2243.0[1817.0, \\
5976.0]\end{array}$ & 0.387 \\
\hline $\operatorname{TnT}(\mathrm{ng} / \mathrm{L})$ & $10.1[2.8,14.7]$ & $12.5[7.2,25.6]$ & 0.236 \\
\hline \multicolumn{4}{|l|}{ CMR findings } \\
\hline LVEDVI $\left(\mathrm{ml} / \mathrm{m}^{2}\right)$ & $150.9 \pm 24.0$ & $158.1 \pm 38.0$ & 0.615 \\
\hline LVEF (\%) & $29.3 \pm 11.2$ & $24.5 \pm 10.1$ & 0.317 \\
\hline LVMI (g) & $70.4 \pm 9.3$ & $76.5 \pm 21.0$ & 0.405 \\
\hline RVEDVI $\left(\mathrm{ml} / \mathrm{m}^{2}\right)$ & $118.8 \pm 38.8$ & $122.5 \pm 44.6$ & 0.845 \\
\hline RVEF (\%) & $34.3 \pm 15.7$ & $33.5 \pm 14.4$ & 0.900 \\
\hline LGE, n (\%) & $2(20)$ & $6(54.5)$ & 0.119 \\
\hline ECV (\%) & $30.7 \pm 2.1$ & $36.8 \pm 5.6$ & 0.005 \\
\hline Native T1 (ms) & $1301 \pm 71$ & $1385 \pm 66$ & 0.011 \\
\hline Post T1 (ms) & $487 \pm 77$ & $418 \pm 40$ & 0.018 \\
\hline T2 (ms) & $40.6 \pm 3.0$ & $43.9 \pm 3.7$ & 0.040 \\
\hline \multicolumn{4}{|l|}{ Medications } \\
\hline ACEI/ARB, n (\%) & $9(90)$ & $11(100)$ & 0.476 \\
\hline$\beta$ blocker, n (\%) & $10(100)$ & $9(81.8)$ & 0.476 \\
\hline Antisterone, n (\%) & $10(100)$ & $11(100)$ & NA \\
\hline Diuretic, n (\%) & $8(80)$ & $10(90.9)$ & 0.586 \\
\hline
\end{tabular}

Abbreviations: $A C E l$ angiotensin-converting enzyme inhibitor, ARB angiotensin II receptor blockers, others are the same as in Table 1 the difference was not statistically significant $(54.5 \%$ vs. $20 \%, P=0.12$ ) (Table 2).

We performed ROC analysis and calculated the sensitivity and specificity of native T1, ECV, and T2 in differentiating the LVEF-recovered and LVEFunrecovered groups. The AUC of ECV for predicting LVEF recovery was 0.83 , with an optimal cutoff value of $32.5 \%$ (sensitivity, $81.8 \%$; specificity, $90.0 \%$ ). The AUC of native T1 for predicting LVEF recovery was 0.80 , with an optimal cutoff value of $1325 \mathrm{~ms}$ (sensitivity, $81.8 \%$; specificity, $70.0 \%$ ). The AUC of T2 for predicting LVEF recovery was 0.76 , and the optimal cutoff value was $43.4 \mathrm{~ms}$ (sensitivity, 63.6\%; specificity, 90.0\%) (Table 3).

In univariate logistic regression analysis, diastolic blood pressure $(\mathrm{OR}=1.15, P=0.045)$, $\mathrm{ECV}(\mathrm{OR}=0.70$, $P=0.029)$, and native $\mathrm{T} 1(\mathrm{OR}=0.98, P=0.030)$ were significantly associated with LV recovery. Systolic blood pressure $(\mathrm{OR}=1.11, P=0.097)$, post-T1 $(\mathrm{OR}=$ 1.02, $P=0.051)$, and T2 $(\mathrm{OR}=0.71, P=0.063)$ tended to be associated with $\mathrm{LV}$ recovery. In multivariate logistic stepwise analysis, only ECV (OR $=0.58,95 \%$ confidence interval $(\mathrm{CI})$ : $0.35-0.96, P=0.032)$ showed an independent association with LV recovery in PPCM patients (Table 4). Figure 2 shows the LGE, native $\mathrm{T} 1, \mathrm{ECV}$, and $\mathrm{T} 2$ images of two patients who did not show LGE, although one patient showed recovered LV function and the other did not show any recovery in LV function.

During the follow-up period (range, 8 months to 5 years; median, 2.5 years), six patients underwent readmission for HF. Among these, one patient showed LVEF recovery after the second readmission and five patients showed no recovery in LVEF. In addition, there were two deaths in the unrecovered group. We performed a Kaplan-Meier survival analysis for MACEs by using the ECV cut-off value of $32.5 \%$. We found that a higher ECV indicated a poor clinical outcome in patients with PPCM $(\log$ rank $P=$ 0.016, Fig. 3).
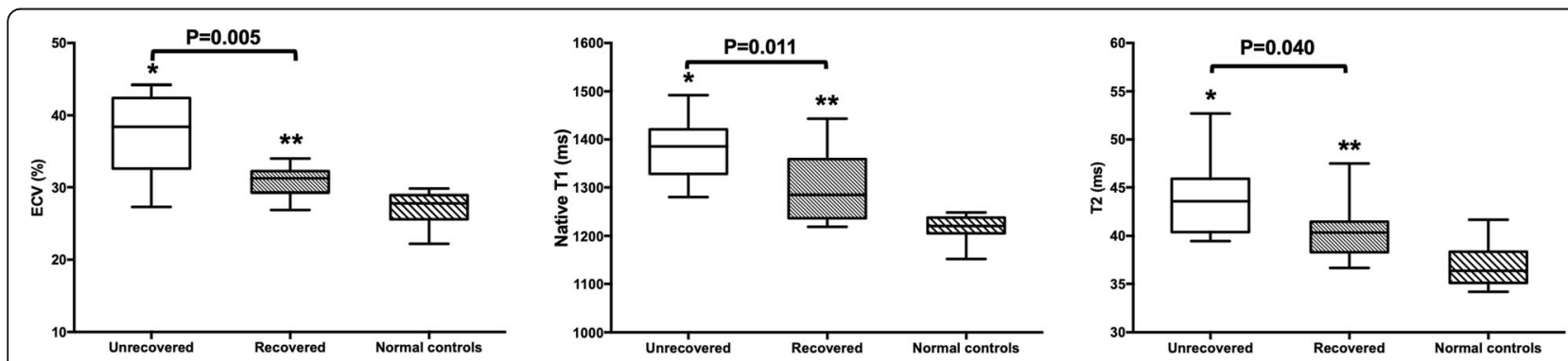

Fig. 1 Comparison of native T1/T2 mapping results for the peripartum cardiomyopathy (PPCM) patients and control groups. ECV, extracellular volume. The center line in each box represents the median, whereas the whiskers show the minimum and maximum values. ${ }^{*} P<0.05$, unrecovered group vs. normal controls; ${ }^{* *} P<0.05$, recovered group vs. normal controls 
Table $3 \mathrm{ROC}$ analysis for quantitative tissue characteristics to differentiate between LVEF recovered and unrecovered PPCM patients

\begin{tabular}{lllll}
\hline Parameters & AUC & Cut-off value & Sensitivity & Specificity \\
\hline ECV (\%) & 0.827 & 32.5 & 0.818 & 0.900 \\
Native T1 (ms) & 0.800 & 1325.1 & 0.818 & 0.700 \\
T2 (ms) & 0.759 & 43.4 & 0.636 & 0.900 \\
\hline
\end{tabular}

Abbreviations: $R O C$ receiver operating characteristic, $A U C$ area under the curve; others are the same as in Table 1

\section{Subgroup analysis of the tissue characteristics in patients with follow-up CMR imaging}

Ten patients $(27.3 \pm 4.9$ years $)$ underwent follow-up CMR imaging (median imaging interval 15.3 months, interquartile range: 12.9 to 22.8 months). At baseline, the results of the subgroup were similar to those of all the subjects. Follow-up CMR had a significantly lower native T1 $(1367 \pm 72$ vs. $1289 \pm 74 \mathrm{~ms}, P=0.019$ by paired T-test). However, there were no significant differences in ECV and T2 between the CMR imaging performed at baseline and at follow-up. Due to the limited sample size, i.e., five LVEF-recovered patients and five other patients, no significant difference was noted in any variable by $\mathrm{T} 1$ and $\mathrm{T} 2$ mapping at baseline and followup CMR imaging (Table 5).

\section{Intra- and inter-observer reproducibility}

Both intra-observer and inter-observer reproducibility for assessments of ECV (intra: intraclass correlation [ICC], 0.96 [0.90 to 0.98]; coefficient of variation [COV], 3.01\%; inter: ICC, 0.96 [0.90 to 0.98]; COV, 3.20\%), native T1 (intra: ICC, 0.96 [0.90 to 0.98]; COV, 1.15\%; inter: ICC, 0.94 (0.86 to 0.98); COV, $1.87 \%$ ), and T2 (intra: ICC, 0.99 [0.97 to 0.99]; COV, 1.20\%; inter: ICC, 0.97 [0.93 to 0.99]; COV, 1.63\%) were excellent.

\section{Discussion}

To the best of our knowledge, this is the first study reporting quantitative $\mathrm{T} 1$ and $\mathrm{T} 2$ mapping in PPCM patients. Our findings suggest that CMR native T1 and T2 mapping may be imaging markers with prognostic potential in PPCM. ECV shows an independent association with LV function recovery in PPCM patients.

In our cohort, none of the 21 PPCM patients had hypertension or preeclampsia. Prior studies have reported that hypertension and preeclampsia are risk factors for PPCM $[10,25]$. However, a recent review of PPCM indicated that preeclampsia or pregnancy-induced hypertension could also trigger pulmonary edema in the absence of PPCM [1]. Furthermore, one recent study directly compared PPCM with hypertension-associated heart failure in

Table 4 Variables associated with the likelihood of left ventricular recovery in PPCM patients by logistic regression

\begin{tabular}{|c|c|c|c|c|c|c|c|c|}
\hline \multirow[t]{3}{*}{ Variables } & \multicolumn{4}{|c|}{ Univariable analysis } & \multicolumn{4}{|c|}{ Multivariable analysis $^{a}$} \\
\hline & \multirow[t]{2}{*}{$\overline{O R}$} & \multicolumn{2}{|l|}{$95 \% \mathrm{Cl}$} & \multirow{2}{*}{$\begin{array}{l}P \\
\text { values }\end{array}$} & \multirow[t]{2}{*}{$\overline{\mathrm{OR}}$} & \multicolumn{2}{|l|}{$95 \% \mathrm{Cl}$} & \multirow{2}{*}{$\begin{array}{l}P \\
\text { values }\end{array}$} \\
\hline & & Lower & $\overline{\text { Upper }}$ & & & Lower & Upper & \\
\hline Age (years) & 0.947 & 0.813 & 1.103 & 0.483 & & & & \\
\hline BMI $\left(\mathrm{kg} / \mathrm{m}^{2}\right)$ & 1.022 & 0.822 & 1.271 & 0.843 & & & & \\
\hline $\mathrm{SBP}(\mathrm{mmHg})$ & 1.108 & 0.982 & 1.250 & 0.097 & 1.216 & 0.989 & 1.496 & 0.064 \\
\hline $\mathrm{DBP}(\mathrm{mmHg})$ & 1.145 & 1.003 & 1.307 & 0.045 & & & & \\
\hline HR (beat/min) & 1.022 & 0.966 & 1.082 & 0.446 & & & & \\
\hline NYHA class & 0.672 & 0.181 & 2.502 & 0.554 & & & & \\
\hline $\operatorname{logNT}$-proBNP (pg/ml) & 0.408 & 0.069 & 2.429 & 0.325 & & & & \\
\hline $\log T n T(n g / L)$ & 0.129 & 0.008 & 2.108 & 0.151 & & & & \\
\hline LVEDVI $\left(\mathrm{ml} / \mathrm{m}^{2}\right)$ & 0.992 & 0.964 & 1.021 & 0.596 & & & & \\
\hline LVEF (\%) & 1.047 & 0.960 & 1.141 & 0.301 & & & & \\
\hline LVMli (g) & 0.975 & 0.919 & 1.033 & 0.392 & & & & \\
\hline RVEDVI $\left(\mathrm{ml} / \mathrm{m}^{2}\right)$ & 0.998 & 0.976 & 1.019 & 0.835 & & & & \\
\hline RVEF (\%) & 1.004 & 0.946 & 1.066 & 0.893 & & & & \\
\hline LGE, n (\%) & 0.208 & 0.030 & 1.467 & 0.115 & & & & \\
\hline ECV (\%) & 0.703 & 0.512 & 0.965 & 0.029 & 0.579 & 0.351 & 0.955 & 0.032 \\
\hline Native T1 (ms) & 0.982 & 0.966 & 0.998 & 0.030 & & & & \\
\hline Post T1 (ms) & 1.024 & 1.000 & 1.048 & 0.051 & & & & \\
\hline $\mathrm{T} 2$ (ms) & 0.708 & 0.492 & 1.019 & 0.063 & & & & \\
\hline
\end{tabular}

$\mathrm{Cl}$ confidence interval, OR odds ratio. Other abbreviations are the same as in Table 1

${ }^{a}$ All covariates with a $P$ value of less than .10 (except for DBP, to avoid the collinearity with SBP) in the univariable analysis were entered into the multivariable model by forward stepwise method 


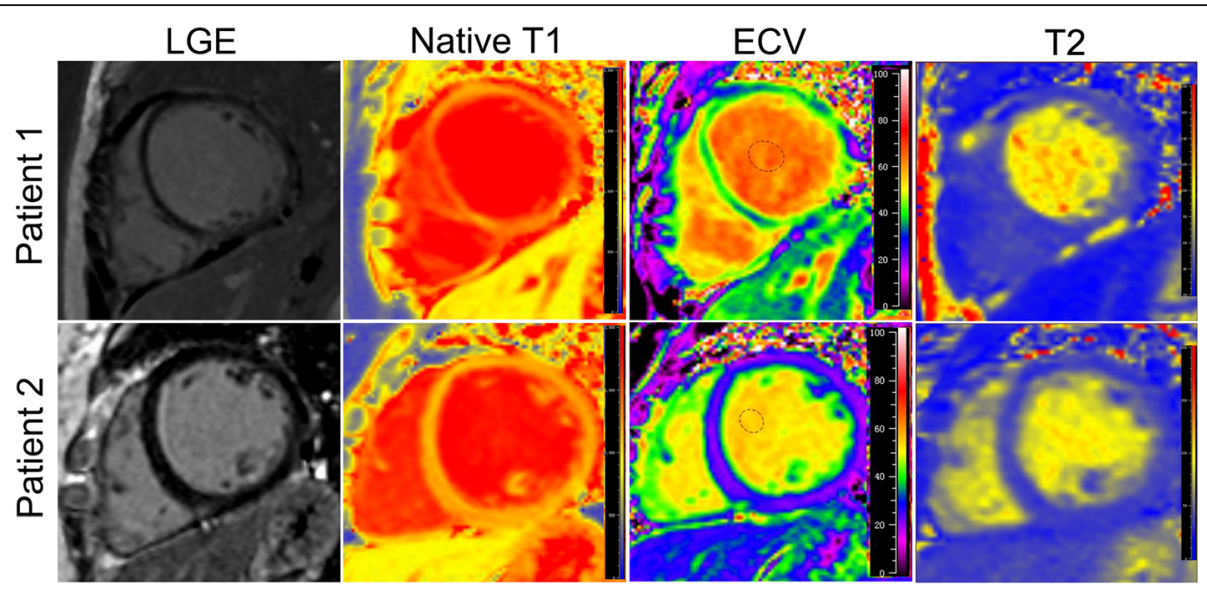

Fig. 2 Cardiovascular magnetic resonance (CMR) images for peripartum cardiomyopathy patients. Patient 1, negative late gadolinium enhancement (LGE), native T1 of 1492 ms, extracellular volume (ECV) of 42.8\%, T2 of 39.5 ms, unrecovered left ventricular ejection fraction (LVEF); Patient 2, negative LGE, native T1 of $1238 \mathrm{~ms}$, ECV of $26.9 \%$, T2 of $36.7 \mathrm{~ms}$, recovered LVEF

pregnancy (HHFP): the results showed that HHFP was associated with different clinical characteristics (cardiac hypertrophy, a preserved $\mathrm{EF}$, and a better prognosis), indicating that the presence of hypertension in pregnancyassociated HF may not indicate PPCM [26]. We are uncertain why none of the patients in our study had hypertension, unless it is related to racial differences [10], the small sample size, or other unknown reasons, although all 21 cases met all of the diagnostic criteria for PPCM.

Unlike previous studies in which the initial LVEF was reported to be a good predictor of LVEF recovery [10, $27,28]$, we did not find that the LVEF-recovered group had significantly higher initial LVEFs than those in the unrecovered group. Previous studies have shown that PPCM is associated with higher rates of thromboembolism than other forms of cardiomyopathy [29, 30]. In addition, many studies have shown that a ventricular thrombus in a PPCM patient is an indicator of poor recovery of LV function [31, 32]. One of the two patients with LV thrombi in our study had unrecovered LV function. Although another patient eventually showed LV recovery, she was readmitted due to signs and symptoms of HF.

In the few previously published CMR studies of PPCM with limited sample sizes, the presence of LGE was likely to predict poor clinical outcomes $[14,15,19]$, while our

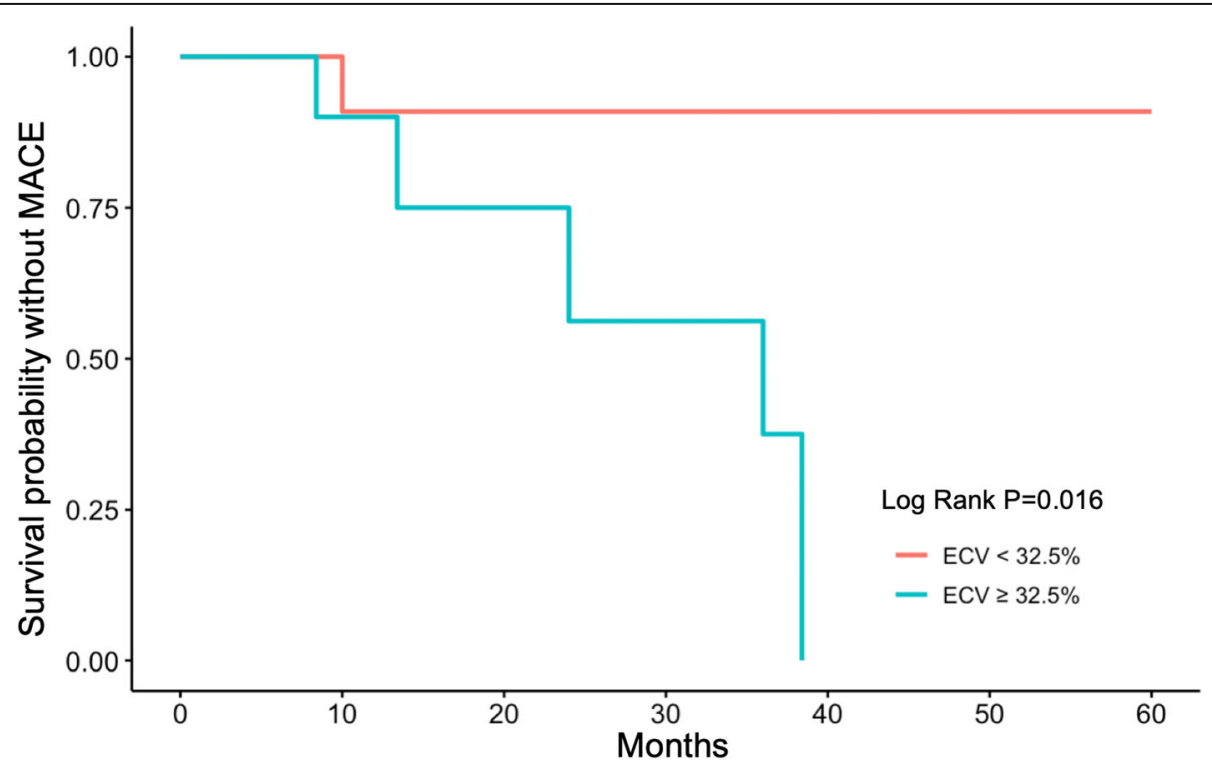

Fig. 3 Kaplan-Meier analysis for extracellular volume in clinical outcomes of patients with peripartum cardiomyopathy. ECV, extracellular volume; MACE, major adverse cardiac event, defined as readmission due to heart failure or cardiac death 
Table 5 The tissue characteristics of PPCM patients by T1 and T2 mapping at baseline and follow-up CMR

\begin{tabular}{|c|c|c|c|c|}
\hline & All PPCM $(n=10)$ & Recovered $(n=5)$ & Unrecovered $(n=5)$ & $P$ values \\
\hline \multicolumn{5}{|l|}{ Baseline CMR } \\
\hline ECV (\%) & $31.9 \pm 4.9$ & $29.8[27.2,32.6]$ & $35.0[28.1,39.1]$ & 0.421 \\
\hline Native T1 (ms) & $1367 \pm 72$ & $1359[13106,1409]$ & $1420[1281,1444]$ & 0.690 \\
\hline Post T1 (ms) & $451 \pm 70$ & $459[400,557]$ & $447[370,475]$ & 0.421 \\
\hline $\mathrm{T} 2$ (ms) & $41.8 \pm 3.0$ & $39.8[38.4,43.8]$ & $42.5[40.6,45.3]$ & 0.310 \\
\hline \multicolumn{5}{|l|}{ Follow-up CMR } \\
\hline ECV (\%) & $30.0 \pm 5.8$ & $29.4[24.1,34.1]$ & $28.4[26.0,36.8]$ & 0.841 \\
\hline Native T1 (ms) & $1289 \pm 74^{*}$ & $1240[1205,1293]$ & $1328[1273,1388]$ & 0.056 \\
\hline Post T1 (ms) & $432 \pm 71$ & $438[395,507]$ & $449[3400,476]$ & 0.841 \\
\hline $\mathrm{T} 2$ (ms) & $40.9 \pm 2.8$ & $40.1[37.1,44.1]$ & $42.1[40.0,42.6]$ & 0.548 \\
\hline \multicolumn{5}{|c|}{ Changes between baseline and follow-up CMR } \\
\hline$\triangle \mathrm{ECV}(\%)$ & $-2.1[-7.1,4.3]$ & $0.3[-7.6,5.8]$ & $-2.4[-7.6,1.2]$ & 0.548 \\
\hline$\Delta$ Native $T 1$ (ms) & $-65[-125,-12]$ & $-110[-2010,-24]$ & $-23[-111,12]$ & 0.222 \\
\hline$\triangle$ Post T1 (ms) & $1.2[-81.6,26.6]$ & $1.0[-80.8,15.3]$ & $2.7[-83.9,53.9]$ & 0.690 \\
\hline$\Delta \mathrm{T} 2$ (ms) & $-0.6[-3.5,0.9]$ & $-0.5[-3.2,2.5]$ & $-2.4[-3.6,1.4]$ & 0.690 \\
\hline
\end{tabular}

${ }^{*} P<0.05$, follow-up CMR vs baseline CMR by paired T-test. Delta values are the differences between those parameters' follow-up value minus baseline value. Abbreviations are the same as in Table 1

study showed that eight of the 21 PPCM patients had LGE and the unrecovered group had a higher percentage of LGE. However, a recent multicenter study of 34 PPCM patients did not demonstrate the value of focal non-ischemic LGE, which occurred in $71 \%$ of the study population [33]. In contrast, another multicenter cohort study reported a very low prevalence of LGE (only 5\%) among 40 PPCM patients [16]. Compared to LGE, the T1 mapping technique may show better performance in quantifying the degree of extracellular matrix or interstitial expansion, particularly in pathologies where the differences between normal and affected myocardium are less obvious [34]. Native T1 can be obtained without the use of gadolinium-based contrast, while ECV can detect early fibrosis changes not always detectable by LGE [35]. Previous studies have shown that native T1 and ECV have good predictive value in dilated cardiomyopathy $[34,36]$, perhaps better than LGE quantification or midwall LGE [37]. Both native T1 and ECV correlated well with the histological collagen volume, which may provide reproducible information on diffuse fibrosis [38]. PPCM and idiopathic dilated cardiomyopathy share some genetic [39] and clinical characteristics, and thus, $\mathrm{T} 1$ mapping might be adopted in a similar scenario. We evaluated ECV in PPCM, and the unrecovered group showed a significantly higher value. Moreover, ECV showed an independent association with the LV recovery in PPCM. In addition, Kaplan-Meier analysis revealed that patients with a higher ECV were at a higher risk of MACEs. A recent CMR study with T1 mapping showed that during normal pregnancy, there is no elevated native T1 [21]. In our study, LV native T1 was obviously higher than that in normal controls, which indicated that the myocardial characteristics had changed in PPCM. Regarding the subgroup analysis of the 10 PPCM patients who underwent follow-up CMR imaging, a significant decrease was noted in the dynamic change in native $\mathrm{T} 1$ at followup. This may indicate an improvement in the myocardial tissue characteristics of PPCM patients. However, due to the small sample size, only a trend rather than statistical significance was observed between LV-recovered and unrecovered groups among those who underwent serial CMR imaging. Further studies on the dynamic changes in myocardial tissue characteristics with a larger sample size are needed.

PPCM is considered to be a form of cardiomyopathy accompanied by inflammation and oxidative stress [40-42]. One case series of CMR findings in PPCM showed that myocardial inflammation in the acute stage was demonstrated by the T2 ratio [18], whereas another case series showed no elevation of the T2 ratio in the acute stage, with only 1 of 10 follow-up images showing an elevated $\mathrm{T} 2$ ratio [14]. We also found $\mathrm{T} 2$ evaluations according to T2 mapping were significantly higher, indicating the presence of edema, in the PPCM group than in the control group. Due to the inherent limitations of the T2 weighted-image method, T2 mapping is preferred to identify edema of the myocardium [20]. Meanwhile, we found that the LVEF-unrecovered group had a higher T2 than the recovered group. This indicates that the degree of inflammation may be associated with PPCM prognosis.

Previous studies have suggested that gamma globulin has a therapeutic effect on the improvement of LVEF in PPCM patients [42]; however, the exact 
efficacy of gamma globulin remains controversial [43]. In the present study, we treated PPCM patients with the guideline-directed medical therapy for HF patients, which includes ACEI/ARB, $\beta$-blockers, aldosterone, diuretics, and warfarin. Since immunoglobulins were not included in the current study, we could not evaluate their efficacy. In addition, we believe that an early risk assessment of whether a PPCM patient might relapse or not mainly helps doctors identify patients who need more long-term follow-up and greater clinical attention. Hence, predictors of CMR imaging are of great significance for clinical decision-making and communicating with PPCM patients and their families.

\section{Limitations}

This study has several limitations. First, the small sample size may preclude conclusions or extrapolations to a wider patient population. Second, due to ethical concerns, age-matched healthy females were chosen as the control group, rather than women with a normal pregnancy. Third, not all CMR examinations were performed in the acute phase, which may affect $\mathrm{T} 2$ value measurement. A more comprehensive assessment of the role of CMR in PPCM through a large, multicenter prospective study with acute-phase and follow-up CMR examinations is needed.

\section{Conclusions}

Native T1, ECV, and T2 were higher in PPCM patients than in normal controls.Our results suggest that native $\mathrm{T} 1, \mathrm{ECV}$, and T2 may be imaging markers with prognostic utility in PPCM. ECV is associated independently with $\mathrm{LV}$ recovery in PPCM patients.

\begin{abstract}
Abbreviations
ACEl: Angiotensin converting enzyme inhibitor; ARB: Angiotensin II receptor blocker; AUC: Area under the curve; bSSFP: Balanced steady state free procession; CMR: Cardiovascular magnetic resonance;

ECG: Electrocardiogram; ECV: Extracellular volume; HCT: Hematocrit; HF: Heart failure; HHFP: Hypertension-associated heart failure in pregnancy; LGE: Late gadolinium enhancement; LV: Left ventricle/left ventricular; LVEDVI: Left ventricular end diastolic volume index; LVEF: Left ventricular ejection fraction; LVMI: Left ventricular mass index; MACE: Major adverse cardiac event; MOLLI: Modified Look-Locker inversion recovery; NT-proBNP: N-terminal proB-type natriuretic peptide; PPCM: Peripartum cardiomyopathy; ROC: Receiver operating characteristic; RV: Right ventricle/right ventricular; RVEDVI: Right ventricular end diastolic volume index; RVEF: Right ventricular ejection fraction; SCMR: Society for Cardiovascular Magnetic Resonance; TI: Inversion time
\end{abstract}

\section{Acknowledgements}

Not applicable.

\section{Authors' contributions}

CYC designed the study. LYD and XYW conducted the study and drafted the manuscript. LWH, WK, and LJY collected the data and undertook the followup work. SJY and ZQ were responsible for the statistical analysis and provided a literature review. CYC and ZXY revised the manuscript. All authors have read and approved the final version of the manuscript.

\section{Authors' information}

CYC (MD, FSCMR, FACC) is a professor in the Department of Cardiology, West China Hospital, Sichuan University, No. 37, Guo Xue Xiang, Chengdu, Sichuan, 610041, China. LYD (MD) is a postdoctoral scholar in the Department of Cardiology, Beijing Hospital, National Center of Gerontology, No.1, Dahua Road, Dongcheng District, Beijing, 100730, China.

Funding

This study is supported by the National Natural Science Foundation of China (Grant No. 81571638).

Availability of data and materials

Data will be available upon request from the corresponding author.

Ethics approval and consent to participate

The Ethics Committee of West China Hospital of Sichuan University approved this study, and informed consent was obtained from each participant.

\section{Consent for publication}

Not applicable.

\section{Competing interests}

The authors declare that they have no competing interests.

\section{Author details}

${ }^{1}$ Department of Cardiology, West China Hospital, Sichuan University, No.37, Guo Xue Xiang, Chengdu, Sichuan 610041, People's Republic of China.

${ }^{2}$ Department of Cardiology, Beijing Hospital, National Center of Gerontology, Beijing, China. ${ }^{3}$ Chinese Academy of Medical Sciences and Peking Union Medical College, Beijing, China. ${ }^{4}$ Department of Geriatrics, West China Hospital, Sichuan University, Chengdu, Sichuan, China. ${ }^{5}$ Department of Radiology, West China Hospital, Sichuan University, Chengdu, Sichuan, China. ${ }^{6}$ MR Collaboration, Siemens Healthineers Ltd., Shanghai, China.

Received: 1 August 2019 Accepted: 5 December 2019

Published online: 06 January 2020

\section{References}

1. Arany Z, Elkayam U. Peripartum cardiomyopathy. Circulation. 2016;133(14): 1397-409.

2. Hilfiker-Kleiner D, Sliwa K. Pathophysiology and epidemiology of peripartum cardiomyopathy. Nat Rev Cardiol. 2014;11(6):364-70.

3. Fett JD, Christie LG, Carraway RD, Ansari AA, Sundstrom JB, Murphy JG. Unrecognized peripartum cardiomyopathy in Haitian women. Int J Gynaecol Obstet. 2005;90(2):161-6.

4. Huang GY, Zhang LY, Long-Le MA, Wang LX. Clinical characteristics and risk factors for peripartum cardiomyopathy. Afr Health Sci. 2012;12(1):26-31.

5. Desai D, Moodley J, Naidoo D. Peripartum cardiomyopathy: experiences at King Edward VIII Hospital, Durban, South Africa and a review of the literature. Trop Dr. 1995;25(3):118-23.

6. Brar SS, Khan SS, Sandhu GK, Jorgensen MB, Parikh N, Hsu JW, et al. Incidence, mortality, and racial differences in peripartum cardiomyopathy. Am J Cardiol. 2007;100(2):302-4.

7. Pearson GD, Veille JC, Rahimtoola S, Hsia J, Oakley CM, Hosenpud JD, et al. Peripartum cardiomyopathy: National Heart, Lung, and Blood Institute and Office of Rare Diseases (National Institutes of Health) workshop recommendations and review. JAMA. 2000:283(9):1183-8.

8. Honigberg MC, Givertz MM. Peripartum cardiomyopathy. BMJ. 2019;364:k5287.

9. Ntusi NB, Mayosi BM. Aetiology and risk factors of peripartum cardiomyopathy: a systematic review. Int J Cardiol. 2009;131(2):168-79.

10. McNamara DM, Elkayam U, Alharethi R, Damp J, Hsich E, Ewald G, et al. Clinical outcomes for peripartum cardiomyopathy in North America: results of the IPAC study (Investigations of Pregnancy-Associated Cardiomyopathy). J Am Coll Cardiol. 2015;66(8):905-14.

11. Barone-Rochette G, Rodiere M, Lantuejoul S. Value of cardiac MRI in peripartum cardiomyopathy. Arch Cardiovasc Dis. 2011;104(4):263-4

12. Elkayam U, Tummala PP, Rao K, Akhter MW, Karaalp IS, Wani OR, et al. Maternal and fetal outcomes of subsequent pregnancies in women with peripartum cardiomyopathy. N Engl J Med. 2001;10(6):1567-71. 
13. Sliwa K, Hilfiker-Kleiner D, Petrie MC, Mebazaa A, Pieske B, Buchmann E, et al. Current state of knowledge on aetiology, diagnosis, management, and therapy of peripartum cardiomyopathy: a position statement from the Heart Failure Association of the European Society of Cardiology Working Group on peripartum cardiomyopathy. Eur J Heart Fail. 2010;12(8):767-78.

14. Arora NP, Mohamad T, Mahajan N, Danrad R, Kottam A, Li T, et al. Cardiac magnetic resonance imaging in peripartum cardiomyopathy. Am J Med Sci. 2014;347(2):112-7.

15. Marmursztejn J, Vignaux O, Goffinet F, Cabanes L, Duboc D. Delayedenhanced cardiac magnetic resonance imaging features in peripartum cardiomyopathy. Int J Cardiol. 2009;137(3):e63-4

16. Schelbert EB, Elkayam U, Cooper LT, Givertz MM, Alexis JD, Briller J, et al. Myocardial damage detected by late gadolinium enhancement cardiac magnetic resonance is uncommon in peripartum cardiomyopathy. J Am Heart Assoc. 2017;6(4)

17. Ersboll AS, Bojer AS, Hauge MG, Johansen M, Damm P, Gustafsson F, et al. Long-term cardiac function after peripartum cardiomyopathy and preeclampsia: a Danish nationwide, clinical follow-up study using maximal exercise testing and cardiac magnetic resonance imaging. J Am Heart Assoc. 2018;7(20):e008991.

18. Renz DM, Rottgen R, Habedank D, Wagner M, Bottcher J, Pfeil A, et al. New insights into peripartum cardiomyopathy using cardiac magnetic resonance imaging. RoFo. 2011;183(9):834-41.

19. Cannan C, Weeks S, Friedrich M. CMR features of peri-partum cardiomyopathy. J Cardiovasc Magn Reson. 2010;12(Suppl 1):1.

20. Giri S, Chung YC, Merchant A, Mihai G, Rajagopalan S, Raman SV, et al. T2 quantification for improved detection of myocardial edema. J Cardiovasc Magn Reson. 2009;11(1):1-13.

21. Nii M, Ishida M, Dohi K, Tanaka H, Kondo E, Ito M, et al. Myocardial tissue characterization and strain analysis in healthy pregnant women using cardiovascular magnetic resonance native T1 mapping and feature tracking technique. J Cardiovasc Magn Reson. 2018;20(1):52.

22. Kramer CM, Barkhausen J, Flamm SD, Kim RJ, Nagel E. Standardized cardiovascular magnetic resonance (CMR) protocols 2013 update. J Cardiovasc Magn Reson. 2013;15:91.

23. Schulz-Menger J, Bluemke DA, Bremerich J, Flamm SD, Fogel MA, Friedrich $M G$, et al. Standardized image interpretation and post processing in cardiovascular magnetic resonance: Society for Cardiovascular Magnetic Resonance (SCMR) board of trustees task force on standardized post processing. J Cardiovasc Magn Reson. 2013;15:35.

24. Arheden $H$, Saeed M, Higgins CB, Gao DW, Bremerich J, Wyttenbach R, et al. Measurement of the distribution volume of gadopentetate dimeglumine at echo-planar MR imaging to quantify myocardial infarction: comparison with 99mTc-DTPA autoradiography in rats. Radiology. 1999;211(3):698-708.

25. Bello N, Rendon IS, Arany Z. The relationship between pre-eclampsia and peripartum cardiomyopathy: a systematic review and meta-analysis. J Am Coll Cardiol. 2013:62(18):1715-23.

26. Ntusi NB, Badri M, Gumedze F, Sliwa K, Mayosi BM. Pregnancy-associated heart failure: a comparison of clinical presentation and outcome between hypertensive heart failure of pregnancy and idiopathic peripartum cardiomyopathy. PLoS One. 2015;10(8):e0133466.

27. Goland S, Bitar F, Modi K, Safirstein J, Ro A, Mirocha J, et al. Evaluation of the clinical relevance of baseline left ventricular ejection fraction as a predictor of recovery or persistence of severe dysfunction in women in the United States with peripartum cardiomyopathy. J Card Fail. 2011;17(5):426-30.

28. Haghikia A, Podewski E, Libhaber E, Labidi S, Fischer D, Roentgen P, et al. Phenotyping and outcome on contemporary management in a German cohort of patients with peripartum cardiomyopathy. Basic Res Cardiol. 2013; 108(4):366.

29. Desplantie O, Tremblay-Gravel M, Avram R, Marquis-Gravel G, Ducharme A, Jolicoeur EM. The medical treatment of new-onset peripartum cardiomyopathy: a systematic review of prospective studies. Can J Cardiol. 2015;31(12):1421-6.

30. Kane A, Mbaye M, Ndiaye MB, Diao M, Moreira PM, Mboup C, et al. Evolution and thromboembolic complications of the idiopathic peripartal cardiomyopathy at Dakar University Hospital: forward-looking study about 33 cases. J Gynecol Obstet Biol Reprod (Paris). 2010;39(6):484-9.

31. Guder G, Brenner S, Morr AK, Frantz S, Angermann CE, Stork S. Serial cardiac imaging in peripartum cardiomyopathy. Int J Cardiol. 2012;157(2):308-9.

32. Garg J, Palaniswamy C, Lanier GM. Peripartum cardiomyopathy: definition, incidence, etiopathogenesis, diagnosis, and management. Cardiol Rev. 2015; 23(2):69-78
33. Haghikia A, Rontgen P, Vogel-Claussen J, Schwab J, Westenfeld R, Ehlermann $\mathrm{P}$, et al. Prognostic implication of right ventricular involvement in peripartum cardiomyopathy: a cardiovascular magnetic resonance study. ESC Heart Fail. 2015;2(4):139-49.

34. Puntmann VO, Carr-White G, Jabbour A, Yu CY, Gebker R, Kelle S, et al. T1mapping and outcome in nonischemic cardiomyopathy: all-cause mortality and heart failure. J Am Coll Cardiol Img. 2016;9(1):40-50.

35. Moon JC, Messroghli DR, Kellman P, Piechnik SK, Robson MD, Ugander M, et al. Myocardial T1 mapping and extracellular volume quantification: a Society for Cardiovascular Magnetic Resonance (SCMR) and CMR Working Group of the European Society of Cardiology consensus statement. J Cardiovasc Magn Reson. 2013;15:92.

36. Barison A, Del Torto A, Chiappino S, Aquaro GD, Todiere G, Vergaro G, et al. Prognostic significance of myocardial extracellular volume fraction in nonischaemic dilated cardiomyopathy. J Cardiovasc Med (Hagerstown). 2015;16(10):681-7.

37. Youn JC, Hong YJ, Lee HJ, Han K, Shim CY, Hong GR, et al. Contrastenhanced T1 mapping-based extracellular volume fraction independently predicts clinical outcome in patients with non-ischemic dilated cardiomyopathy: a prospective cohort study. Eur Radiol. 2017;27:3924-3933.

38. Nakamori S, Dohi K, Ishida M, Goto Y, Imanaka-Yoshida K, Omori T, et al. Native T1 mapping and extracellular volume mapping for the assessment of diffuse myocardial fibrosis in dilated cardiomyopathy. J Am Coll Cardiol Img. 2017:11:48-59.

39. Ware JS, Li J, Mazaika E, Yasso CM, DeSouza T, Cappola TP, et al. Shared genetic predisposition in peripartum and dilated cardiomyopathies. N Engl J Med. 2016:374(3):233-41.

40. Fett JD. Inflammation and virus in dilated cardiomyopathy as indicated by endomyocardial biopsy. Int J Cardiol. 2006;112(1):125-6.

41. Ansari AA, Fett JD, Carraway RE, Mayne AE, Onlamoon N, Sundstrom JB. Autoimmune mechanisms as the basis for human peripartum cardiomyopathy. Clin Rev Allergy Immunol. 2002;23(3):301-24.

42. Bozkurt B, Villaneuva FS, Holubkov R, Tokarczyk T, Alvarez RJ Jr, MacGowan $G A$, et al. Intravenous immune globulin in the therapy of peripartum cardiomyopathy. J Am Coll Cardiol. 1999;34(1):177-80.

43. Lindley KJ, Verma AK, Blauwet LA. Peripartum cardiomyopathy: progress in understanding the etiology, management, and prognosis. Heart Fail Clin. 2019;15(1):29-39.

\section{Publisher's Note}

Springer Nature remains neutral with regard to jurisdictional claims in published maps and institutional affiliations.
Ready to submit your research? Choose BMC and benefit from:

- fast, convenient online submission

- thorough peer review by experienced researchers in your field

- rapid publication on acceptance

- support for research data, including large and complex data types

- gold Open Access which fosters wider collaboration and increased citations

- maximum visibility for your research: over $100 \mathrm{M}$ website views per year

At $\mathrm{BMC}$, research is always in progress.

Learn more biomedcentral.com/submissions 